\title{
Sleeping Difficulty and Subjective Short Sleep Duration are Associated with Serum N-terminal Pro-brain Natriuretic Peptide Levels in the Elderly Population
}

\author{
Nobuo Sasaki ${ }^{1}$, Hideya Yamamoto ${ }^{2}$, Ryoji Ozono ${ }^{3}$, Ryo Maeda $^{1}$ and Yasuki Kihara ${ }^{4}$
}

\begin{abstract}
:
Objective It is well known that poor sleep increases the risk of heart failure (HF). However, the underlying mechanisms remain unclear. In this study, we investigated the association of poor sleep with hemodynamic stress on the left ventricle, which was a key factor for the development of HF in elderly individuals.

Methods A total of 2,301 participants ( $\geq 65$ years old) without cardiac disease were enrolled in this crosssectional analysis. We evaluated the subjective sleep quality, sleeping difficulty, subjective sleep duration, use of sleeping pills, and daytime dysfunction using the Pittsburgh Sleep Quality Index, a 19-item self-reported questionnaire. We assessed serum N-terminal pro-brain natriuretic peptide (NT-proBNP) as a marker of hemodynamic stress on the left ventricle, and we defined high NT-proBNP as a serum NT-proBNP level $\geq$ $125 \mathrm{pg} / \mathrm{mL}$.

Results Sleeping difficulty was significantly associated with high NT-proBNP levels [odds ratio (OR), 1.46; 95\% confidence interval $(\mathrm{CI}), 1.16-1.85 ; \mathrm{p}<0.005]$. A subjective short sleep duration was also significantly associated with high NT-proBNP levels (OR, 1.69; $95 \%$ CI, 1.03-2.75; p<0.05). A subjective poor sleep quality, the use of sleeping pills, and daytime dysfunction were not associated with serum NT-proBNP levels. All data were adjusted for the age, sex, body mass index, serum hemoglobin concentration, serum creatinine level, systolic blood pressure, diastolic blood pressure, and use of antihypertensive medications.

Conclusion Poor sleep was associated with high hemodynamic stress to the left ventricle in elderly population.
\end{abstract}

Key words: elderly, heart failure, natriuretic peptide, brain, sleep

(Intern Med 59: 2213-2219, 2020)

(DOI: 10.2169/internalmedicine.4470-20)

\section{Introduction}

Sleep problems, such as difficulty initiating and maintaining sleep, increase with age, and a large proportion of the elderly population suffer from poor sleep (1). Although some studies have reported that poor sleep increases the risk for heart failure (HF) (2-4), the underlying mechanisms remain unclear. Several experimental studies have reported that both a poor sleep quality and short sleep duration may cause activation of the sympathetic nervous system and the hypothalamic-pituitary-adrenal axis (5-8), elevation of blood pressure (9-13), and development of obesity by affecting the levels of hunger-inducing hormones $(14,15)$. All of these may increase hemodynamic stress to the left ventricle, which plays a critical role in the development of heart failure in the elderly population (16). However, few studies have investigated the association between poor sleep and hemodynamic stress to the left ventricle.

$\mathrm{N}$-terminal pro-brain natriuretic peptide (NT-proBNP) is

${ }^{1}$ Health Management and Promotion Center, Hiroshima Atomic Bomb Casualty Council, Japan, ${ }^{2}$ Laboratory of Physiology and Morphology, School of Pharmacy, Yasuda Women's University, Japan, ${ }^{3}$ Department of General Medicine, Hiroshima University Graduate School of Biomedical and Health Sciences, Japan and ${ }^{4}$ Department of Cardiovascular Medicine, Hiroshima University Graduate School of Biomedical and Health Sciences, Japan

Received: January 12, 2020; Accepted: March 11, 2020; Advance Publication by J-STAGE: June 15, 2020

Correspondence to Dr. Nobuo Sasaki,nb7ssk7@sd6.so-net.ne.jp 


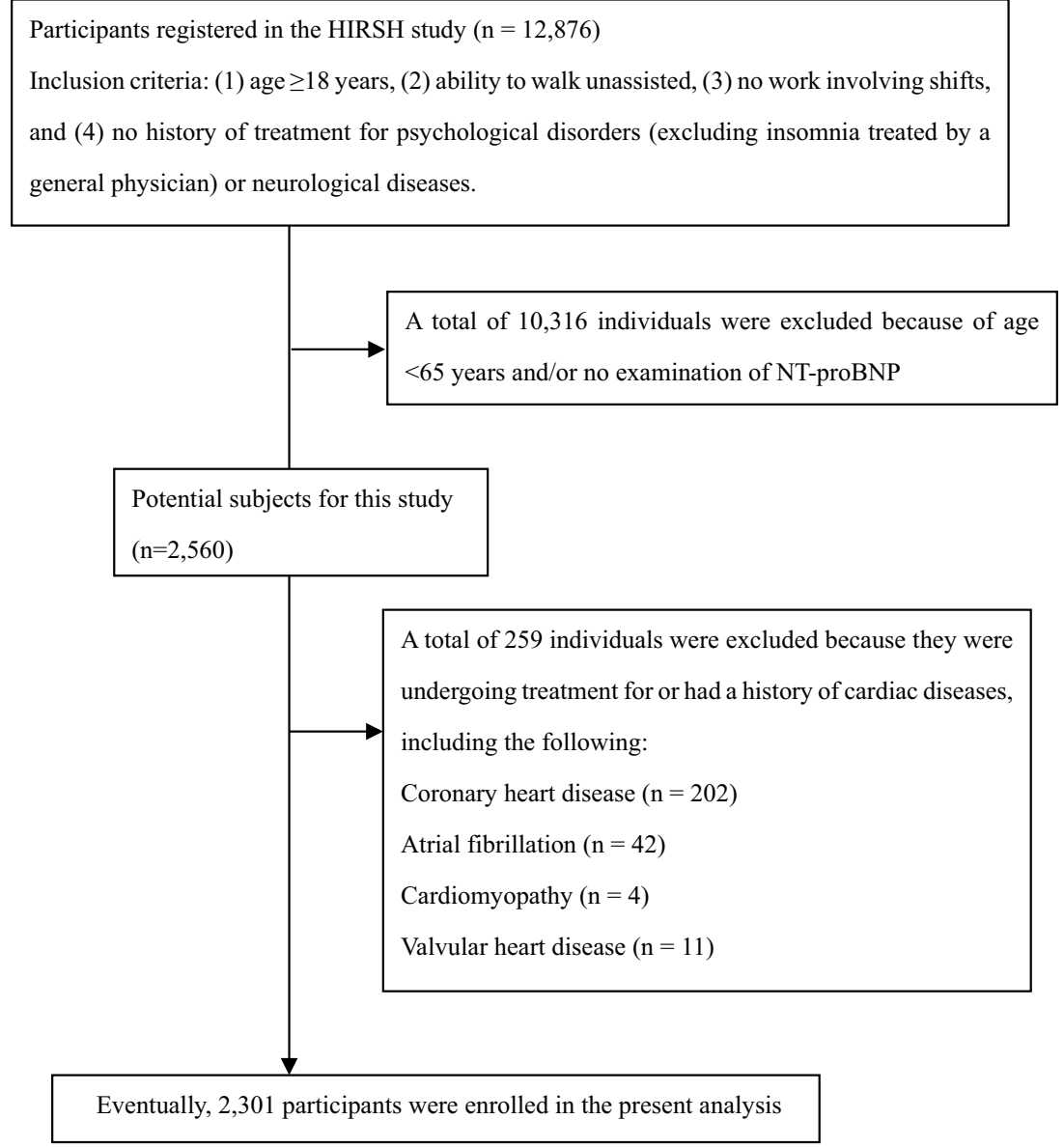

Figure 1. Flowchart for the selection of participants. HIRSH study, Hiroshima Sleep and Healthcare study; NT-proBNP: N-terminal pro-brain natriuretic peptide

an established marker of HF (17). The serum level of this cardiac hormone reflects hemodynamic stress to the left ventricle in people without cardiac disease (18), because NTproBNP is released from the myocardium in response to increased cardiac wall stress from both volume and pressure overload (19).

In this study, we investigated the association of the selfreported sleep quality with the serum NT-proBNP level using the Pittsburgh Sleep Quality Index (PSQI) in elderly individuals without cardiac disease.

\section{Materials and Methods}

\section{Study participants and design}

In November 2014, we commenced the Hiroshima Sleep and Healthcare (HIRSH) study (20-22), which was an observational study that assessed the association of sleep habits with lifestyle-related diseases. Potential participants were recruited from among residents of Hiroshima City and Mihara City who were undergoing annual health examinations at the Health Management and Promotion Center of the Hiroshima Atomic Bomb Casualty Council or cooperating facilities. The inclusion criteria for the HIRSH study were as follows: (1) $\geq 18$ years old, (2) able to walk unassisted, (3) no work involving shifts, and (4) no history of treatment for psychological disorders (excluding insomnia treated by a general physician) or neurological diseases.

A total of 12,876 individuals were registered in the HIRSH study between November 2014 and September 2015. Of these individuals, 2,560 potential participants were selected according to the following criteria: (1) $\geq 65$ years old and (2) data from an optional screening check for serum NT-proBNP levels at the baseline examination in the HIRSH study available. Among these participants, 259 were excluded because they were undergoing treatment for or had a history of cardiac diseases, including coronary heart disease $(n=202)$, atrial fibrillation $(n=42)$, cardiomyopathy $(n=4)$, and valvular heart disease $(n=11)$. Thus, a total of 2,301 participants (1,051 men and 1,250 women) with complete data were eventually included in the present analysis.

The mean \pm standard deviation (SD) of the age was $75.7 \pm$ 5.3 years, and the mean $\pm \mathrm{SD}$ of the body mass index (BMI) was $22.9 \pm 3.1 \mathrm{~kg} / \mathrm{m}^{2}$ (Fig. 1). Of these participants, $958(42 \%)$ were taking antihypertensive medications, 298 (13\%) had diabetes mellitus (defined as taking antidiabetic medications), 583 (25\%) had dyslipidemia (defined as taking antihyperlipidemic medications), 130 (6\%) had a history of stroke, $178(8 \%)$ were current smokers (defined as having a current smoking habit, regardless of the number of cigarettes 
smoked per day), and 689 (30\%) were habitual drinkers (defined as drinking alcohol for $\geq 5$ days per week, regardless of the amount consumed).

Written informed consent was obtained from all participants. The study protocol conformed to the ethical guidelines of the 1975 Declaration of Helsinki and was approved by the Hiroshima Atomic Bomb Casualty Council committee.

\section{Measurements}

The sleep quality was evaluated using the Japanese version of the PSQI $(23,24)$. The PSQI is a 19-item selfreported questionnaire used to assess the degree of sleep problems over the previous month and contains a 7component scale (each component is ranked $0,1,2$ or 3 ), including the following factors: subjective sleep quality (C1), sleep latency (C2), subjective sleep duration (C3), habitual sleep efficiency (C4), sleep disturbances (C5), use of sleeping pills (C6) and daytime dysfunction (C7). Based on each PSQI component, we defined a subjective poor sleep quality, short sleep duration, use of sleeping pills, and daytime dysfunction as a $\mathrm{C} 1$ score $\geq 2$, a C3 score $\geq 3$, a C6 score $\geq 1$, and a $\mathrm{C} 7$ score $\geq 1$, respectively. Furthermore, we defined sleeping difficulty (i.e., difficulty initiating and/or maintaining sleep) as the sum of the $\mathrm{C} 2, \mathrm{C} 4$, and $\mathrm{C} 5$ scores $\geq 4$. A C2 score that was calculated from the results of two different types of questions was interpreted as difficulty initiating sleep, and a C5 score that was calculated from answers to nine detailed questions regarding trouble sleeping was interpreted as difficulty maintaining sleep. The C4 score was calculated using the following formula: (total sleep time / time in bed) $\times 100$. The sum of the $\mathrm{C} 2, \mathrm{C} 4$, and C5 scores provides a comprehensive and effective assessment of what is defined as sleeping difficulty.

We collected blood samples from all participants to measure the hemoglobin concentration and serum levels of creatinine and NT-proBNP. NT-proBNP was measured by a chemiluminescent enzyme immunoassay using a Cobas e601 analyser (Roche Diagnostics, Tokyo, Japan) (25). High NTproBNP was defined as a serum NT-proBNP level $\geq 125 \mathrm{pg}$ / $\mathrm{mL}$ (26). Using a digital, automatic blood pressure (BP)measuring instrument (Terumo, Tokyo, Japan; or Omron Healthcare, Kyoto, Japan), the BP was measured at the heart level with the participant in a seated position on a chair with back support and arm support after resting for $>5$ minutes while undergoing a health examination.

\section{Statistical analyses}

Continuous variables are expressed as the mean $\pm \mathrm{SD}$ or median (interquartile range, 25-75\%). Categorical variables are summarized as percentages and were analyzed using the chi-square test. The relationships between characteristics of participants and high NT-proBNP levels were evaluated using a univariate logistic regression analysis to calculate the odds ratio (OR) and 95\% confidence interval (CI). The associations of the sleep quality (overall poor sleep, subjective poor sleep quality, sleeping difficulty, subjective short sleep duration, use of sleeping medications, and daytime dysfunction) with high NT-proBNP levels were assessed using a multivariate logistic regression analysis. The model included age, female sex (yes or no) (27), BMI, serum hemoglobin concentration (28), serum creatinine level (29), systolic BP, diastolic BP, and the use of antihypertensive medications (yes or no). All of these were variables with $\mathrm{p}$ values $<0.05$ in the univariate logistic regression analysis (Model 1). Next, the multivariate model simultaneously included sleeping difficulty and subjective short sleep duration (Model 2). $\mathrm{P}$ values $<0.05$ were considered statistically significant. All statistical analyses were performed using the JMP 10 statistical software program (SAS Institute, Cary, USA).

\section{Results}

Participant characteristics and the results of the PSQI are shown in Table 1. The median (interquartile range, 25-75\%) NT-proBNP level was $78.0 \mathrm{pg} / \mathrm{mL}$ (range, 46.0-136.0 pg/ $\mathrm{mL}$ ), and the proportion of all participants with high NTproBNP levels was 29\%. The proportions of participants with a subjective poor sleep quality, sleeping difficulty, subjective short sleep duration, use of sleeping medications, and daytime dysfunction were $19 \%, 22 \%, 4 \%, 21 \%$, and $22 \%$, respectively. Supplementary material 1 shows the association between serum NT-proBNP levels and each component of PSQI including $\mathrm{C} 1$ to $\mathrm{C} 7$.

The fraction of participants with high NT-proBNP levels among those with and without poor sleep is featured in Fig. 2. NT-proBNP levels were significantly higher in participants with sleeping difficulties than in those without sleeping difficulties $(37.0 \%$ vs. $26.6 \%, \mathrm{p}<0.001)$; NTproBNP levels were also significantly higher in those with subjective short sleep duration than in those without subjective short sleep duration $(39.5 \%$ vs. $28.4 \%, \mathrm{p}=0.031)$.

Table 2 shows the results of the univariate logistic regression analysis. The age, gender, BMI, serum creatinine level, hemoglobin concentration, systolic blood pressure, diastolic blood pressure, and use of antihypertensive medications were significantly associated with high NT-pro BNP levels.

After adjusting for confounding factors, sleeping difficulty was significantly associated with high NT-proBNP levels (OR, 1.46; 95\% CI, 1.16-1.85; p<0.005). A subjective short sleep duration was also significantly associated with high NT-proBNP levels (OR, 1.69; 95\% CI, 1.03-2.75; p<0.05; see Model 1 in Table 3). After adjusting for the presence of sleep difficulty, the significance of the association between a subjective short sleep duration and high NT-proBNP levels was lost (Model 2 in Table 3).

\section{Discussion}

We investigated the association of poor sleep with serum NT-proBNP levels in elderly individuals without cardiac disease. A subjective poor sleep quality, use of sleeping pills, 
Table 1. Characteristics and Results of Pittsburgh Sleep Quality Index in Participants.

\begin{tabular}{|c|c|c|}
\hline $\mathrm{N}$ & & 2,301 \\
\hline Mean age & (years) & $75.7 \pm 5.3$ \\
\hline Female & {$[\mathrm{n}(\%)]$} & $1,250(54)$ \\
\hline BMI & $\left(\mathrm{kg} / \mathrm{m}^{2}\right)$ & $22.9 \pm 3.1$ \\
\hline Hemoglobin & $(\mathrm{g} / \mathrm{dL})$ & $13.3 \pm 1.4$ \\
\hline Creatinine & $(\mathrm{mg} / \mathrm{dL})$ & $0.79 \pm 0.21$ \\
\hline SBP & $(\mathrm{mmHg})$ & $133 \pm 17$ \\
\hline DBP & $(\mathrm{mmHg})$ & $74 \pm 11$ \\
\hline Current smoker & [n (\%)] & $178(8)$ \\
\hline Habitual drinker & {$[\mathrm{n}(\%)]$} & $689(30)$ \\
\hline Antihypertensive medications & {$[\mathrm{n}(\%)]$} & $958(42)$ \\
\hline Diabetes & {$[\mathrm{n}(\%)]$} & $298(13)$ \\
\hline Dyslipidaemia & {$[\mathrm{n}(\%)]$} & $583(25)$ \\
\hline History of stroke & {$[\mathrm{n}(\%)]$} & $130(6)$ \\
\hline NT-proBNP ${ }^{\dagger}$ & $(\mathrm{pg} / \mathrm{mL})$ & $78(46-136)$ \\
\hline High NT-proBNP & {$[\mathrm{n}(\%)]$} & $664(29)$ \\
\hline Global PSQI score & & $5.1 \pm 3.2$ \\
\hline C1 score & & $1.0 \pm 0.6$ \\
\hline C2 score & & $0.9 \pm 0.9$ \\
\hline C3 score & & $1.1 \pm 0.9$ \\
\hline C4 score & & $0.6 \pm 0.9$ \\
\hline C5 score & & $0.7 \pm 0.5$ \\
\hline C6 score & & $0.5 \pm 1.1$ \\
\hline C7 score & & $0.2 \pm 0.5$ \\
\hline Subjective poor sleep quality & {$[\mathrm{n}(\%)]$} & 442 (19) \\
\hline Sleeping difficulty & {$[\mathrm{n}(\%)]$} & $495(22)$ \\
\hline Short sleep duration & {$[\mathrm{n}(\%)]$} & $86(4)$ \\
\hline Use of sleeping pills & {$[\mathrm{n}(\%)]$} & $483(21)$ \\
\hline Daytime dysfunction & {$[\mathrm{n}(\%)]$} & 495 (22) \\
\hline
\end{tabular}

Hypertension was defined as taking anti-hypertensive medications and/or having systolic blood pressure $\geq 140 \mathrm{mmHg}$ and/or diastolic blood pressure $\geq 90 \mathrm{mmHg}$. Subjective poor sleep quality, sleep difficulty, short sleep duration, use of sleeping pills, and daytime dysfunction were defined as $\mathrm{C} 1$ score $\geq 2$, the sum of $\mathrm{C} 2, \mathrm{C} 4$ and C5 scores $\geq 4$, C3 score $\geq 3$, C6 score $\geq 1$, and C7 score $\geq 1$, respectively. The scores from $\mathrm{C} 1$ to $\mathrm{C} 7$ were components of PSQI. High NT-proBNP was defined as serum NT-proBNP level $\geq 125$ $\mathrm{pg} / \mathrm{mL}$. $\dagger$ Data are given as median (interquartile range, $25-75 \%$ ). BMI: body mass index, DBP: diastolic blood pressure, NT-proBNP: N-terminal pro-brain natriuretic peptide, PSQI: Pittsburgh Sleep Quality Index, SBP: systolic blood pressure

and daytime dysfunction were not associated with the serum NT-proBNP levels. Conversely, sleeping difficulty and a subjective short sleep duration were associated with the serum NT-proBNP levels, suggesting that these elements of poor sleep may have significant hemodynamic impacts on the left ventricle in elderly individuals without cardiac disease.

In this study, we evaluated sleeping difficulty as the sum of the C2, C4, and C5 scores of the PSQI components, which reflect sleep latency, sleep efficiency, and sleep fragmentation, respectively. Zhang et al. reported that a longer sleep latency and lower sleep efficiency were correlated with higher 24-hour urinary catecholamine levels in healthy subjects (30). Several experimental studies showed that sleep fragmentation increases sympathetic nervous activity, adrenocortical activity, and $\operatorname{BP}(7,11,12)$. Chouchou et al. reported that increased frequency of arousal, a marker of sleep fragmentation, was associated with an increase in sympathetic nervous activity based on a large population observational study (31). Taken together, these findings suggest that sleeping difficulty, including longer sleep latency, sleep fragmentation and resultant lower sleep efficiency, may increase hemodynamic stress to the left ventricle through activation of the sympathetic nervous system and the hypothalamic-pituitary-adrenal axis and elevation of BP.

In a laboratory setting, sleep deprivation also caused activation of the sympathetic nervous system (5), elevation of blood pressure (9) and risk of weight gain $(14,15)$, all of which can increase the hemodynamic stress to the left ventricle. In this study, we defined subjective short sleep duration as a $\mathrm{C} 3$ score $\geq 3$ (equivalent to $<5$ hours), which is an extremely short duration compared with the recommended sleep duration for optimal health (32). One possible explanation for the association between a subjective short sleep duration and a high NT-proBNP level is that a subjective short sleep duration may have similar effects on the heart to those of sleep deprivation in experimental studies $(5,9)$. However, after adjusting for the presence of sleep difficulty, the significance of the association between a subjective short sleep duration and high NT-proBNP level was lost. Thus, the impact of a subjective short sleep duration on the heart may depend on the sleep quality, as a long sleep latency, sleep fragmentation, and low sleep efficiency usually result in a short sleep duration.

A number of studies have reported that poor sleep increases the risk for coronary heart disease and stroke (33-35). In contrast, available data regarding the association between poor sleep and HF are limited. Laugsand et al. reported that the number of insomnia symptoms (difficulty initiating sleep, difficulty maintaining sleep, and having nonrestorative sleep) was associated with incident HF (2). Wannamethee et al. reported that self-reported daytime sleep longer than 1 hour, which suggested poor sleep during the night, was associated with an increased risk of HF in elderly men and that a self-reported short sleep duration ( $<6$ hours) was associated with an increased risk of HF in elderly men with pre-existing cardiovascular disease (3). Ingelsson et al. reported that sleep disturbance (difficulty falling asleep and/or waking up in the early hours and/or taking sleeping pills) was associated with HF risk in participants with obesity (defined as BMI $>25 \mathrm{~kg} / \mathrm{m}^{2}$ ) (4). However, these studies did not suggest potential mechanisms underlying the link between poor sleep and HF. In the present study, we showed a significant hemodynamic linkage between poor sleep and the left ventricle, which may be involved in the development of HF. Recently, Zonoozi et al. reported that daytime sleep duration $>1$ hour was associated with a increased risk of elevated NT-proBNP levels in elderly men (36). This is partly consistent with our results, as daytime sleep may correlate with poor sleep during the 


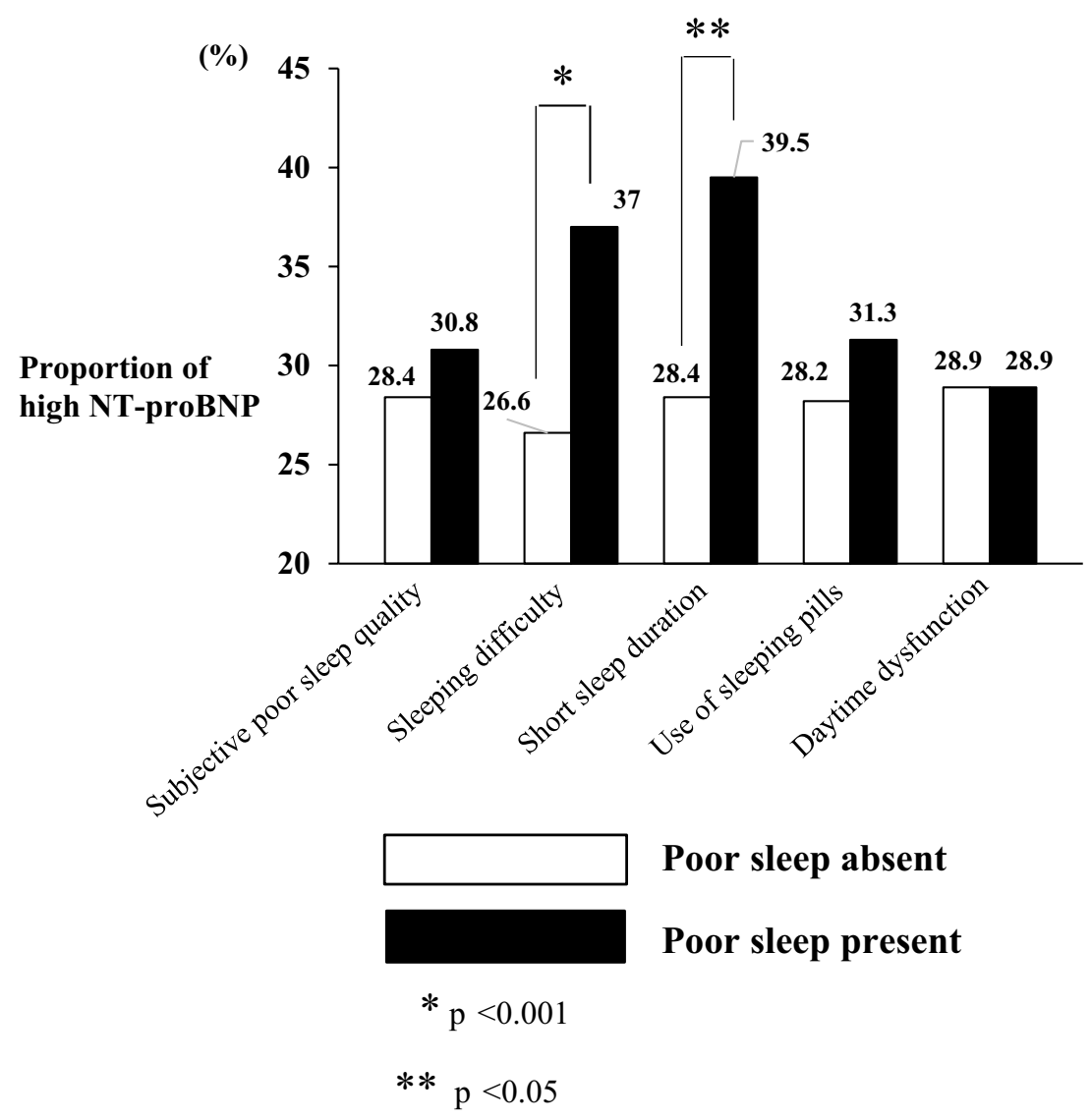

Figure 2. A comparison of the proportion of participants with high NT-proBNP levels between those with and without poor sleep. A subjective poor sleep quality, sleep difficulty, short sleep duration, use of sleeping pills and daytime dysfunction were defined as $\mathrm{C} 1$ score $\geq 2$; the sum of $\mathrm{C2}, \mathrm{C4}$, and $\mathrm{C} 5$ scores $\geq 4$; $\mathrm{C} 3$ score $\geq 3$; C6 score $\geq 1$; and $\mathrm{C} 7$ score $\geq 1$, respectively. The scores from $\mathrm{C} 1$ to C7 were components of the PSQI. High NT-proBNP was defined as a serum NT-proBNP level $\geq 125$ pg/mL. NT-proBNP: N-terminal pro-brain natriuretic peptide, PSQI: Pittsburgh Sleep Quality Index

Table 2. Univariate OR for High NT-proBNP $(n=2,301)$.

\begin{tabular}{lccc}
\hline \multicolumn{1}{c}{ Variable } & OR & $95 \% \mathrm{CI}$ & $\mathrm{p}$ \\
\hline Age, years & 1.14 & $(1.12-1.16)$ & $<0.001$ \\
Female, yes or no & 1.24 & $(1.04-1.49)$ & 0.019 \\
BMI, kg/m & 0.94 & $(0.91-0.97)$ & $<0.001$ \\
Hemoglobin, g/dL & 0.63 & $(0.58-0.68)$ & $<0.001$ \\
Creatinine, mg/dL & 4.57 & $(2.99-7.04)$ & $<0.001$ \\
SBP, mmHg & 1.01 & $(1.01-1.02)$ & $<0.001$ \\
DBP, mmHg & 0.98 & $(0.97-0.99)$ & $<0.001$ \\
Current smoker, yes or no & 0.8 & $(0.55-1.12)$ & 0.198 \\
Habitual drinker, yes or no & 0.98 & $(0.81-1.19)$ & 0.855 \\
Antihypertensive medications, yes or no & 1.56 & $(1.30-1.87)$ & $<0.001$ \\
Diabetes, yes or no & 0.98 & $(0.75-1.28)$ & 0.892 \\
Dyslipidaemia, yes or no & 0.90 & $(0.73-1.11)$ & 0.327 \\
History of stroke, yes or no & 1.19 & $(0.81-1.73)$ & 0.376 \\
\hline
\end{tabular}

Hypertension was defined as taking anti-hypertensive medications and/or having systolic blood pressure $\geq 140 \mathrm{mmHg}$ and/or diastolic blood pressure $\geq 90 \mathrm{mmHg}$. High NT-proBNP was defined as serum NT-proBNP level $\geq 125 \mathrm{pg} / \mathrm{mL}$. BMI: body mass index, CI: confidence interval, DBP: diastolic blood pressure, NT-proBNP: N-terminal pro-brain natriuretic peptide, OR: odds ratio, SBP: systolic blood pressure 
Table 3. Multivariable Adjusted OR for High NT-proBNP $(n=2,301)$.

\begin{tabular}{lcccccc}
\hline \multirow{2}{*}{ Variable } & \multicolumn{3}{c}{ Model 1 } & \multicolumn{3}{c}{ Model 2 } \\
\cline { 2 - 7 } & OR & $95 \%$ CI & p & OR & $95 \%$ CI & $\mathrm{p}$ \\
\hline Subjective poor sleep quality & 1.18 & $(0.92-1.50)$ & 0.202 & & & \\
Sleeping difficulty & 1.46 & $(1.16-1.85)$ & 0.002 & 1.40 & $(1.10-1.79)$ & 0.007 \\
Short sleep duration & 1.69 & $(1.03-2.75)$ & 0.039 & 1.40 & $(0.83-2.32)$ & 0.204 \\
Use of sleeping medications & 0.91 & $(0.71-1.16)$ & 0.440 & & & \\
Daytime dysfunction & 0.95 & $(0.74-1.21)$ & 0.664 & & & \\
\hline
\end{tabular}

Multivariate model 1 included age, gender, body mass index, serum creatinine, hemoglobin concentration, SBP, DBP and the use of antihypertensive medications. Model 2 simultaneously included sleeping difficulty and short sleep duration.

Subjective poor sleep quality, sleep difficulty, short sleep duration, use of sleeping pills, and daytime dysfunction were defined as $\mathrm{C} 1$ score $\geq 2$, the sum of $\mathrm{C} 2$, C4 and C5 scores $\geq 4$, C3 score $\geq 3$, C6 score $\geq 1$, and $\mathrm{C} 7$ score $\geq 1$, respectively. The scores from $\mathrm{C} 1$ to $\mathrm{C} 7$ were components of PSQI. High NT-proBNP was defined as serum NT-proBNP level $\geq 125 \mathrm{pg} / \mathrm{mL}$. CI: confidence interval, DBP: diastolic blood pressure, NT-proBNP: N-terminal pro-brain natriuretic peptide, OR: odds ratio, PSQI: Pittsburgh Sleep Quality Index, SBP: systolic blood pressure

night.

Several previous studies have reported that the use of sleeping pills may have adverse effects on health, including an increased risk for cardiovascular disease $(37,38)$. However, little is known about whether or not the use of sleeping pills increases the risk of HF. Our results suggest that the use of sleeping pills was not associated with a hemodynamic impact on the left ventricle in elderly individuals without cardiac disease.

In the present study, the assessment of sleep was based on a self-reported questionnaire. Some studies have suggested that self-reported questionnaires may provide crude estimates of sleep habits compared with objective methods (39). Indeed, sleep duration evaluated by objective methods is more accurate than that by self-reported questionnaires. However, it is well known that the amount of sleep required by a person varies from individual to individual (32). The measurement by objective methods does not include the evaluation whether the amount of sleep is sufficient for sleep needed in the individual. One merit associated with the subjective sleep duration is that it evaluates whether the amount of sleep obtained is enough for a given individual. Several investigators have shown that subjective sleep evaluations are equivalent to objective methods in assessing relationships with pathology $(40,41)$. A large number of studies have revealed that the sleep duration and quality evaluated using a self-reported questionnaire were associated with cardiovascular morbidity and mortality $(42,43)$.

Several limitations associated with the present study warrant mention. First, because of its cross-sectional design, a causal association between the sleep quality and serum NTproBNP levels cannot be inferred. Second, because we did not assess obstructive sleep apnea (OSA), which frequently accompanies a poor sleep quality and cardiac dysfunction (44), we cannot exclude the possibility that OSA was partly involved in the association of a poor sleep quality with high NT-proBNP levels in this study. However, daytime dysfunction was not associated with the serum NT-proBNP level in this study, suggesting that OSA may have a limited impact on our results. This point was critical, as excessive sleepiness was significantly associated with an increased risk of $\mathrm{HF}$ in patients with OSA (45).

In conclusion, our cross-sectional analysis demonstrated that sleeping difficulty and subjective short sleep duration were associated with serum NT-proBNP levels, suggesting a hemodynamic linkage between poor sleep and the left ventricle in elderly individuals. The prevalence of HF, especially HF with a preserved ejection fraction, is increasing with the growing size of the elderly population. Poor sleep may be involved in the development of HF through an increase in hemodynamic stress to the left ventricle in the elderly population.

The authors state that they have no Conflict of Interest (COI).

\section{Acknowledgement}

The authors thank Naomi Yuzono for her technical and secretarial assistance.

\section{References}

1. Foley DJ, Monjan AA, Brown SL, Simonsick EM, Wallace RB, Blazer DG. Sleep complaints among elderly persons: an epidemiologic study of three communities. Sleep 18: 425-432, 1995.

2. Laugsand LE, Strand LB, Platou C, Vatten LJ, Janszky I. Insomnia and the risk of incident heart failure: a population study. Eur Heart J 35: 1382-1393, 2014.

3. Wannamethee SG, Papacosta O, Lennon L, Whincup PH. Selfreported sleep duration, napping, and incident heart failure: prospective associations in the British Regional Heart Study. J Am Geriatr Soc 64: 1845-1850, 2016.

4. Ingelsson E, Lind L, Arnlöv J, Sundström J. Sleep disturbances independently predict heart failure in overweight middle-aged men. Eur J Heart Fail 9: 184-190, 2007.

5. Zhong X, Hilton HJ, Gates GJ, et al. Increased sympathetic and decreased parasympathetic cardiovascular modulation in normal humans with acute sleep deprivation. J Appl Physiol 98: 20242032, 2005.

6. Tasali E, Leproult R, Ehrmann DA, Cauter EV. Slow-wave sleep and the risk of type 2 diabetes in humans. Proc Natl Acad Sci 105: 1044-1049, 2008.

7. Stamatakis KA, Punjabi NM. Effects of sleep fragmentation on 
glucose metabolism in normal subjects. Chest 137: 95-101, 2010.

8. Spiegel K, Leproult R, Van Cauter E. Impact of sleep debt on metabolic and endocrine function. Lancet 354: 1435-1439, 1999.

9. Robillard R, Lanfranchi PA, Prince F, Filipini D, Carrier J. Sleep deprivation increases blood pressure in healthy normotensive elderly and attenuates the blood pressure response to orthostatic challenge. Sleep 34: 335-339, 2011.

10. Tochikubo O, Ikeda A, Miyajima E, Ishii M. Effects of insufficient sleep on blood pressure monitored by a new multibiomedical recorder. Hypertension 27: 1318-1324, 1996.

11. Davies RJ, Belt PJ, Roberts SJ, Ali NJ, Stradling JR. Arterial blood pressure responses to graded transient arousal from sleep in normal humans. J Appl Physiol 74: 1123-1130, 1993.

12. Garcia CE, Drager LF, Krieger EM, et al. Arousals are frequent and associated with exacerbated blood pressure response in patients with primary hypertension. Am J Hypertens 26: 617-623, 2013.

13. Sayk F, Teckentrup C, Becker C, et al. Effects of selective slowwave sleep deprivation on nocturnal blood pressure dipping and daytime blood pressure regulation. Am J Physiol Regul Integr Comp Physiol 298: R191-R197, 2010.

14. Spiegel K, Tasali E, Penev P, Van Cauter E. Brief communication: sleep curtailment in healthy young men is associated with decreased leptin levels, elevated ghrelin levels, and increased hunger and appetite. Ann Intern Med 141: 846-850, 2004.

15. Bosy-Westphal A, Hinrichs S, Jauch-Chara K, et al. Influence of partial sleep deprivation on energy balance and insulin sensitivity in healthy women. Obes Facts 1: 266-273, 2008.

16. Lam CS, Gona P, Larson MG, et al. Aortic root remodeling and risk of heart failure in the Framingham Heart study. JACC Heart Fail 1: 79-83, 2013

17. Troughton RW, Frampton CM, Brunner-La Rocca HP, et al. Effect of B-type natriuretic peptide-guided treatment of chronic heart failure on total mortality and hospitalization: an individual patient meta-analysis. Eur Heart J 35: 1559-1567, 2014.

18. Hirata $\mathrm{Y}$, Matsumoto A, Aoyagi T, et al. Measurement of plasma brain natriuretic peptide level as a guide for cardiac overload. Cardiovasc Res 51: 585-591, 2001.

19. Vanderheyden M, Goethals $M$, Verstreken $S$, et al. Wall stress modulates brain natriuretic peptide production in pressure overload cardiomyopathy. J Am Coll Cardiol 44: 2349-2354, 2004.

20. Sasaki N, Ozono R, Yamashita H, et al. Association of sleep habits with blood pressure in elderly people. Clin Exp Hypertens 38: 733-737, 2016.

21. Sasaki N, Fujiwara S, Yamashita H, Ozono R, Teramen K, Kihara Y. Impact of sleep on osteoporosis: sleep quality is associated with bone stiffness index. Sleep Med 25: 73-77, 2016.

22. Sasaki N, Fujiwara S, Yamashita $H$, et al. Association between obesity and self-reported sleep duration variability, sleep timing, and age in the Japanese population. Obes Res Clin Pract 12: 187194, 2018.

23. Buysse DJ, Reynolds CF 3rd, Monk TH, Berman SR, Kupfer DJ. The Pittsburgh Sleep Quality Index: a new instrument for psychiatric practice and research. Psychiatry Res 28: 193-213, 1989.

24. Doi Y, Minowa M, Uchiyama M, et al. Psychometric assessment of subjective sleep quality using the Japanese version of the Pittsburgh Sleep Quality Index (PSQI-J) in psychiatric disordered and control subjects. Psychiatry Res 97: 165-172, 2000.

25. Yeo KT, Wu AH, Apple FS, et al. Multicenter evaluation of the Roche NT-proBNP assay and comparison to the Biosite Triage BNP assay. Clin Chim Acta 338: 107-115, 2003.

26. Doi $\mathrm{Y}$, Ninomiya $\mathrm{T}$, Hata $\mathrm{J}$, et al. $\mathrm{N}$-terminal pro-brain natriuretic peptide and risk of cardiovascular events in a Japanese community: the Hisayama study. Arterioscler Thromb Vasc Biol 31: $2997-$ 3003, 2011.

27. Kim HL, Kim MA, Choi DJ, et al. Gender difference in the prognostic value of $\mathrm{N}$-terminal pro-B type natriuretic peptide in patients with heart failure - a report from the Korean Heart Failure Registry (KorHF). Circ J 81: 1329-1336, 2017.

28. Tanimura M, Dohi K, Fujimoto N, et al. Effect of anemia on cardiovascular hemodynamics, therapeutic strategy and clinical out- comes in patients with heart failure and hemodynamic congestion. Circ J 81: 1670-1677, 2017.

29. Sasaki N, Yamamoto H, Ozono R, Fujiwara S, Kihara Y. Association of N-terminal pro B-type natriuretic peptide with blood pressure and pulse pressure in elderly people: a cross-sectional population study. Circ J 82: 2049-2054, 2018.

30. Zhang J, Ma RC, Kong AP, et al. Relationship of sleep quantity and quality with 24-hour urinary catecholamines and salivary awakening cortisol in healthy middle-aged adults. Sleep 34: 225233, 2011.

31. Chouchou F, Pichot V, Pépin JL, et al. Sympathetic overactivity due to sleep fragmentation is associated with elevated diurnal systolic blood pressure in healthy elderly subjects: the PROOFSYNAPSE study. Eur Heart J 34: 2122-2131, 2013.

32. Watson NF, Badr MS, Belenky G, et al.; Consensus Conference Panel. Joint consensus statement of the American Academy of Sleep Medicine and Sleep Research Society on the recommended amount of sleep for a healthy adult: methodology and discussion. Sleep 38: 1161-1183, 2015.

33. Cappuccio FP, Cooper D, D'Elia L, Strazzullo P, Miller MA. Sleep duration predicts cardiovascular outcomes: a systematic review and meta-analysis of prospective studies. Eur Heart J 32: 1484-1492, 2011.

34. Laugsand LE, Vatten LJ, Platou C, Janszky I. Insomnia and the risk of acute myocardial infarction: a population study. Circulation 124: 2073-2081, 2011

35. Li Y, Zhang X, Winkelman JW, et al. Association between insomnia symptoms and mortality: a prospective study of U.S. men. Circulation 129: 737-746, 2014.

36. Zonoozi S, Ramsay SE, Papacosta O, et al. Self-reported sleep duration and napping, cardiac risk factors and markers of subclinical vascular disease: cross-sectional study in older men. BMJ Open 7: 2017.

37. Petrov ME, Howard VJ, Kleindorfer D, Grandner MA, Molano JR, Howard G. Over-the-counter and prescription sleep medication and incident stroke: the REGARDS study. J Stroke Cerebrovasc Dis 23: $2110-2116,2014$.

38. Thorogood M, Cowen P, Mann J, Murphy M, Vessey M. Fatal myocardial infarction and use of psychotropic drugs in young women. Lancet 340: 1067-1068, 1992.

39. Bathgate CJ, Edinger JD, Wyatt JK, Krystal AD. Objective but not subjective short sleep duration associated with increased risk for hypertension in individuals with insomnia. Sleep 39: 1037-1045, 2016.

40. Van Der Kloet D, Giesbrecht T, Franck E, et al. Dissociative symptoms and sleep parameters-an all-night polysomnography study in patients with insomnia. Compr Psychiatry 54: 658-664, 2013.

41. Meng L, Zheng Y, Hui R. The relationship of sleep duration and insomnia to risk of hypertension incidence: a meta-analysis of prospective cohort studies. Hypertens Res 36: 985-995, 2013.

42. Cappuccio FP, Cooper D, D'Elia L, Strazzullo P, Miller MA. Sleep duration predicts cardiovascular outcomes: a systematic review and meta-analysis of prospective studies. Eur Heart J 32: 1484-1492, 2011.

43. Chien KL, Chen PC, Hsu HC, et al. Habitual sleep duration and insomnia and the risk of cardiovascular events and all-cause death: report from a community-based cohort. Sleep 33: 177-184, 2010.

44. Shahar E, Whitney CW, Redline S, et al. Sleep-disordered breathing and cardiovascular disease: cross-sectional results of the Sleep Heart Health Study. Am J Respir Crit Care Med 163: 19-25, 2001.

45. Mazzotti DR, Keenan BT, Lim DC, Gottlieb DJ, Kim J, Pack AI. Symptom subtypes of obstructive sleep apnea predict incidence of cardiovascular outcomes. Am J Respir Crit Care Med 200: 493506, 2019.

The Internal Medicine is an Open Access journal distributed under the Creative Commons Attribution-NonCommercial-NoDerivatives 4.0 International License. To view the details of this license, please visit (https://creativecommons.org/licenses/ by-nc-nd/4.0/). 